\title{
Zonificación molecular de yacimientos de edad eoceno y mioceno al norte de la cuenca del lago de Maracaibo
}

\author{
Jhoan Jose Urdaneta \\ Corporación Venezolana del Petróleo (CVP) \\ E-mail: Urdanetajjx@gmail.com
}

\begin{abstract}
Resumen
El propósito de esta investigación es documentar la zonificación molecular de los crudos entrampados en la Formación Basal La Rosa (BLR) / Arenas B, localizados en el Bloque I, del Occidente de la República Bolivariana de Venezuela específicamente en la Cuenca del Lago de Maracaibo, en las áreas VLA0016 y VLA0033 de la Unidad de Producción Lagomar. Para el desarrollo del mismo se llevó a cabo la recolección de las muestras de crudo en campo siguiendo la metodología estándar, luego a nivel de laboratorio se determinaron las relaciones atómicas entre compuestos para establecer la transformación físico-química de la materia orgánica desde su depositación hasta su estado actual. Una vez obtenidos el dato de campo se llevó a cabo la inyección de la misma en el equipo de Cromatografía de Gases acoplado a un Espectrómetro de Masa, donde ocurre la separación de los compuestos para su posterior análisis detallado. Los resultados de ésta investigación permiten establecer los niveles de madurez térmica, origen de materia orgánica, huella digital del crudo, ambiente de depositación, alteraciones del crudo a través de relaciones estadísticas entre compuestos, con el fin de caracterizar geoquímicamente el crudo del área de interés.
\end{abstract}

Palabras clave: Huella digital, Biomarcadores, Pristano, Fitano, Diagrama Estrella.

\section{Molecular zoning of eocene and miocene age deposits in the north of the Maracaibo lake basin}

\begin{abstract}
The purpose of this research is to document the molecular zoning of trapped crudes in La Rosa Basal Formation (BLR) / Arenas B, located in Block I, in the West of the Bolivarian Republic of Venezuela, specifically in the Lake Maracaibo Basin, in areas VLA0016 and VLA0033 of the Lagomar Production Unit. For the development of this study, a collection of the crude samples in the field following the standard methodology was carried out. Then, at the laboratory level, the atomic relationships between compounds were determined to establish the physical-chemical transformation of the organic material from its deposition to their current state. Once the field data were obtained, the injection thereof was carried out in the Gas Chromatography equipment coupled to a Mass Spectrometer, where the separation of the compounds took place for their subsequent detailed analysis. The results of this investigation allowed us to establish the levels of thermal maturity, origin of organic material, fingerprint of the crude oil, environment of deposition, alterations of the crude through statistical relationships between compounds, in order to characterize geochemically the crude oil of the area of interest.
\end{abstract}

Keywords: Fingerprint, Biomarkers, Pristane, Phytane, Star Diagram.

\section{Introducción}

La Cuenca del Lago de Maracaibo, posee los campos petrolíferos de más importancia económica para la Región Occidental Venezolana, que están integrados por varios pozos que producen petróleo de yacimientos ubicados en diferentes niveles estratigráficos de edad cretácica y terciaria. Los datos y las muestras que se analizaron y estudiaron fueron muestreados en cabezal de pozo, utilizando la metodología aprendida del Instituto Zuliano de Investigaciones Tecnológicas (INZIT).

Cita: Urdaneta, J. J. (2019). Zonificación molecular de yacimientos de edad eoceno y mioceno al norte de la cuenca del lago de Maracaibo. Revista Fuentes: El reventón energético, 17(1), 37-46. 
Los resultados obtenidos a partir de estos análisis permitieron mostrar la presencia de crudos con distintas características geoquímicas indicando el origen de materia orgánica, ambiente deposicional, grado de madurez, gravedad $\mathrm{API}^{\circ}$ y la posibilidad de existencia de varios procesos de alteración dentro del yacimiento (Figueira et al, 2017). Entre los principales procesos de degradación de un crudo se pueden mencionar: biodegradación, lavado por agua, alteración térmica, entre otros (Navas et al, 2017). También, las diferencias detectadas apuntarían a una falta de comunicación entre varios compartimientos que han sido aislados entre ellos y por consecuencia, han seguido diferentes líneas de evolución estructural y composicional (Castro y Mederos, 2016).

Finalmente, se determinaron los parámetros moleculares de los crudos de interés, a través de análisis de biomarcadores extraídos del crudo por cromatografía de gases acoplado a espectrometría de masa, esto en aras de mitigar la incertidumbre molecular existente entre los yacimientos.

\section{Ubicación Geográfica}

El área de estudio se encuentra localizada al noroeste de la República Bolivariana de Venezuela, dentro de la Cuenca Petrolífera de Maracaibo, la cual posee una extensión estimada de $47.705 \mathrm{~km}^{2}$. Al oeste-noroeste, la cuenca está delimitada por el piedemonte de la Sierra de Perijá; hacia el este-noreste por el piedemonte occidental de la Serranía de Trujillo; al sureste por el piedemonte andino hacia el Río Motatán; al norte e imaginariamente delimitada por una línea en la frontera entre los estados Zulia y Falcón y por la línea geológica de la Falla de Oca-Ancón. Las líneas mencionadas anteriormente son bastante arbitrarias en sentido fisiográfico y geológico, pero corresponden en realidad al carácter geo-económico de la cuenca petrolífera como tal. A nivel local, el estudio fue elaborado en el Bloque I perteneciente a la Unidad de Producción Lagomar del Distrito Maracaibo, el cual se encuentra situado en la parte centro-norte del Lago de Maracaibo, ocupando una extensión de $242.324 \mathrm{Km}^{2}$ y encontrándose dividido en flanco este y flanco oeste. Específicamente, el área de estudio, denominada VLA0016 / VLA0033, cuya dimensión es aproximadamente $11.888 \mathrm{~km}^{2}$, corresponde al Campo Lagunillas y se ubica en la zona centro-norte de El Pilar en el Bloque I del Lago de Maracaibo. El Área VLA0016/VLA0033 limita estructuralmente al oeste por una falla de tipo normal con la Unidad de Producción Urdaneta Lago, hacia el este con la Falla Lama-Icotea, hacia su parte norte con un límite arbitrario de la Unidad de Producción Rosa
Mediano y al sur con dos fallas que se interceptan a nivel del mismo miembro Santa Bárbara. Tabla 2 se muestran las coordenadas UTM que delimitan el área de estudio y en la Ilustración 1 puede apreciarse la ubicación geográfica del Área VLA0016 / VLA0033.

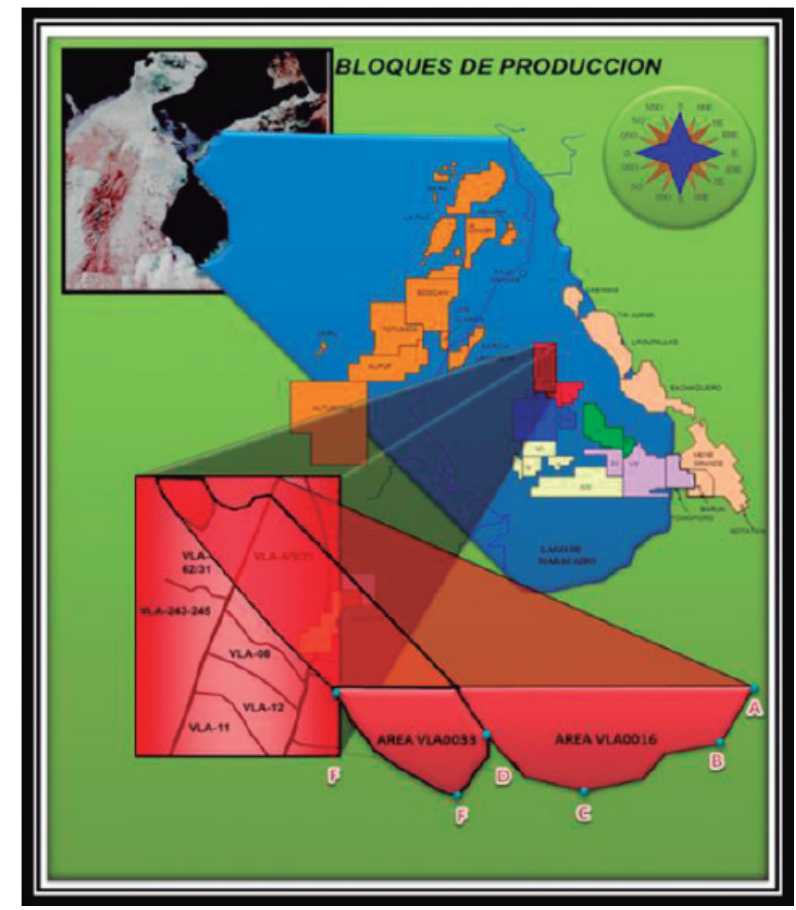

Ilustración 1. Ubicación Geográfica del Área de Estudio. Área VLA0033 / VLA0016 en el Bloque I del Lago de Maracaibo, Venezuela.

\section{Marco Metodológico}

La investigación de esta investigación es de carácter descriptivo e interpretativo. El universo de muestras analizadas fue de 9 pozos distribuidos en las áreas CLA0016 y VLA0033 del Bloque I campo Lagunillas de la Cuenca del Lago de Maracaibo, haciendo énfasis en crudos medianos y livianos del area (9 Pozos), gracias a que en ellos se logró realizar un mayor número de análisis. El mismo involucró la toma de muestras de estos pozos de la Unidad de Producción Lagomar (U.P. Lagomar).

En primer lugar, se procedió a la búsqueda y recopilación de toda la información disponible en cuanto a estudios realizados anteriormente y bibliografía. Las muestras fueron sometidas a análisis SARA (separación de las fracciones de saturados, aromáticos, resinas y asfáltenos), para posteriormente analizar las fracciones de aromáticos y saturados a través de cromatografía de gases acoplada a espectrometría de masa con el fin de cuantificar de manera relativa los biomarcadores 
presentes en el crudo, con el fin de caracterizar y simular los yacimientos (Martin et al, 2014; Guerrero et al, 2018).

\section{Resultados y Discusión}

Los resultados obtenidos en esta investigación, mediante el procesamiento, análisis e interpretación de los datos arrojados por la población de crudos estudiados, se llevaron a cabo a través de estudio geologico del área, y su posterior toma de muestra en cabezal del pozo que seguidamente fueron analizados en laboratorio, a fin de determinar la caracterización geoquímica de los crudos del área.

\section{Características Generales De Los Crudos}

Los crudos marinos de la Cuenca del Lago de Maracaibo, provenientes de pozos completados en distintos depósitos de la columna geológica de esta cuenca, han sido generados en su gran mayoría, por la Formación La Luna.

De acuerdo a la composición general de los crudos (hidrocarburos saturados, hidrocarburos aromáticos y resinas + asfaltenos), el porcentaje de hidrocarburos saturados en relación con la gravedad API, también puede proporcionar tendencias de madurez, entre mayor es el contenido de saturados, disminuyen los aromáticos y las resinas más asfaltenos; de aquí que los crudos se conviertan en crudos más livianos como los de este estudio.

Los análisis geoquímicos realizados sobre las muestras de crudos de yacimientos Terciarios, incluyeron el análisis SARA, la cromatografía de gases acoplada a espectrometría de masas de las fracciones de Saturados y Aromáticos para revisar la concentración y distribución de biomarcadores de estos hidrocarburos (Guardia et al, 2011; Martin, 2017).

\section{Relación de parafinicidad Vs aromaticidad de Thompson (1988)}

La caracterización, correlación y clasificación de los hidrocarburos se establece en función de su origen, grado de madurez alteración dentro del yacimiento como biodegradación, lavado por aguas fraccionamiento evaporativo, y mesclas de hidrocarburos, por esta razón se plantea la metodología propuesta por K. F. Thompson (1988), que se basa en la evidencia de que la fracción ligera de algunos crudos que contienen concentraciones muy altas de compuestos aromáticos y naftenos, mientras que la proporción de compuestos n-alcanos es baja.
Así, se definen los términos aromaticidad (Tolueno/ $\mathrm{n}-\mathrm{C} 7$ ), que se relaciona con fraccionamiento evaporativo, y parafinicidad (n-C7-/Metilciclohexano), valor que se incrementa con la madurez del crudo. El gráfico propuesto por Thompson permite clasificar los crudos livianos y condensados de acuerdo al tipo de alteración: fraccionamiento evaporativo, madurez, lavado por agua y biodegradación.

En este gráfico, el sentido de las flechas indica una tendencia de incremento en el proceso o bien que el proceso es más avanzado, por ejemplo, en los crudos de área de estudio, ellos están siendo afectados, por una alteración de fraccionamiento evaporativo, ubicado en la zona A, por otra parte también muestra una buena madurez, pero conforme se presenta el proceso de fraccionamiento evaporativo los crudos residuales tienden a incrementar la aromaticidad, en la zona A, mientras que si se analiza la fracción ligera migrada se encontraría incremento de la parafinicidad.

\section{Determinación de correlación y transformación de crudos según Halpern (1995).}

Buscando las diferencias en los datos de los compuestos C7- de petróleos inalterados comparados con crudos biodegradados de la misma familia se construyeron las relaciones por interacción a fin de enfatizar las diferencias.

El parámetro Tr1 mide la disminución de tolueno que es el compuesto C7; más soluble en agua, y por to tanto es útil para determinar la alteración (lavado por aguas). Las relaciones restantes miden biodegradación y la susceptibilidad de los compuestos involucrados disminuye en sentido contrario a las manecillas del reloj hacia la relación $\operatorname{Tr} 8$, es decir, la relación más resistente y estable con la biodegradación. El parámetro Tr6 está formado por compuestos que difieren significativamente en los puntos de ebullición, por lo que resulta ser un parámetro más resistente (menos susceptible) a la transformación que los parámetros $\operatorname{Tr}$ menores. Consecuentemente, el parámetro Tr6 es muy útil para medir evaporación causada por el manejo inapropiado de muestras y probablemente por efecto de fraccionamiento-migración, en ambos casos el parámetro incrementara su valor. Por otra parte, el diagrama de estrella para determinar correlaciones entre los petróleos está formado por relaciones que son resistentes o estables a los procesos de transformación y que están relacionados con el origen de los crudos. Esto significa que las relaciones son invariantes virtualmente dentro de una misma familia, pero que muestran cambios significativos entre los crudos de diferentes familias. 


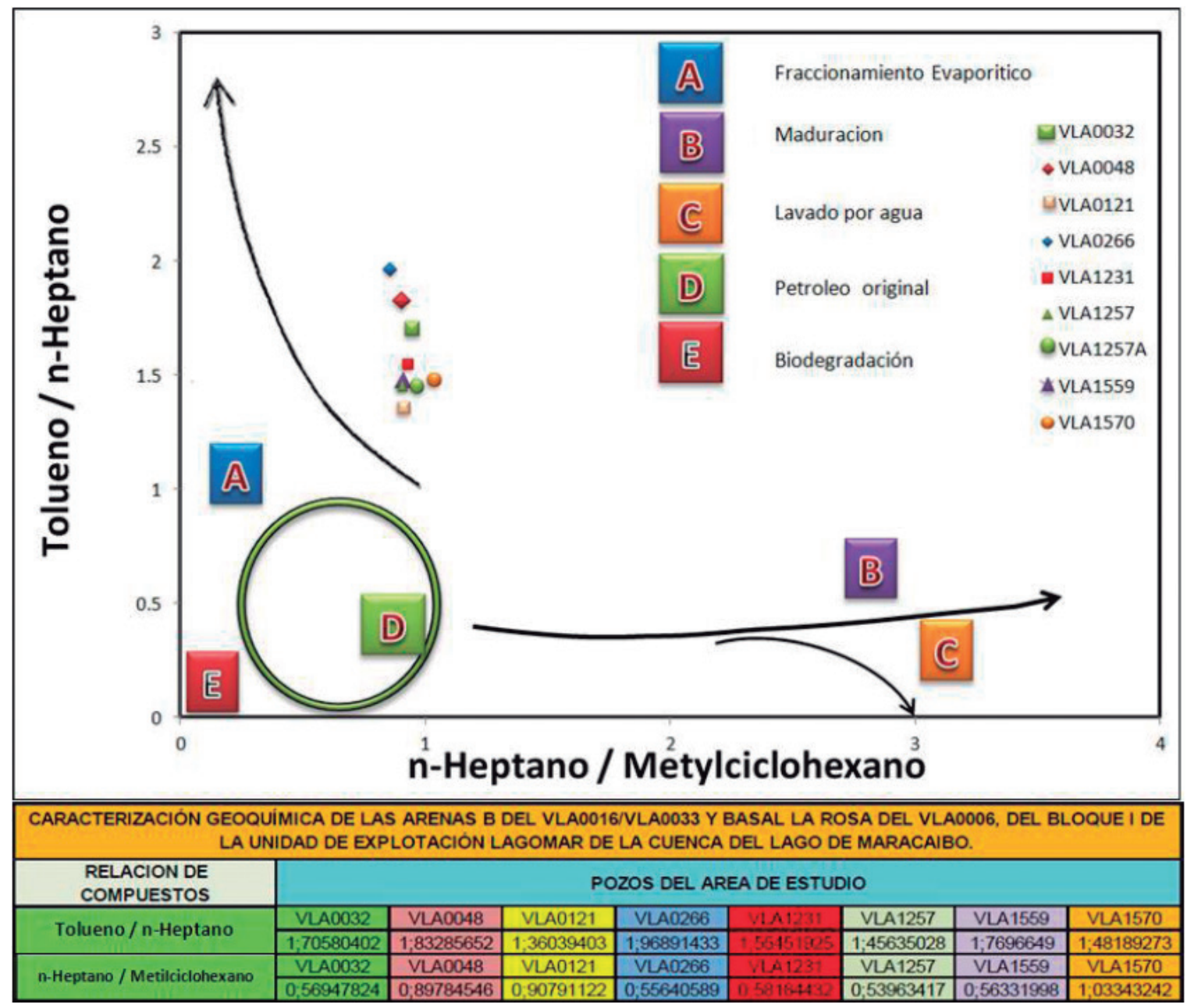

Ilustración 2. Relación de Parafinicidad vs. Aromaticidad.

Las relaciones $\mathrm{C} 1$ a $\mathrm{C} 5$ pueden ser utilizadas para monitorear evaporación o fraccionamiento-migración entre muestras de una misma familia. La relación $\mathrm{C} 1$ tiene una diferencia en temperaturas de ebullición de -6 entre el numerador y el denominador y debe disminuir con la evaporación, mientras que la relación C5 tiene una diferencia de +8 y por to tanto debe incrementarse.

El diagrama estrella para la transformación de crudos con base en el alto porcentaje de tolueno para el compuesto $\mathrm{C} 7$, indica que los crudos en estudio, no presentan alteración de lavado por aguas, de las nueve (9) muestras, solo se diferenciaron los pozos VLA1257, VLA0121, VLA1570 y VLA0048 por presentar un menor valor de tolueno en el pico $\operatorname{Tr} 1$, no siendo afectados por este tipo de alteración. Sin embargo se puedo inferir que el total de las muestras analizadas (8), presentan alteraciones por fraccionamiento evaporativo.
El parámetro Tr6 afecta todos los crudos de los pozos analizados, exceptuando al pozo VLA0266, que presenta un proceso de alteración distinto asociado más a una alteración por fraccionamiento - migración, debido a la resistencia que opone con respecto al resto de los crudos.

El diagrama estrella para determinar correlaciones de crudo según lo expuesto por Halpern (1988), manifiesta que las muestras en estudios exhiben un perfil similar, aunado a ello, se tiene que para el parámetro $\mathrm{C} 1$ en todos los crudos a excepción de los pozos VLA0266 y VLA1570, difieren de los otros, siendo esto un indicativo de un posible fraccionamiento - migración, en donde otros autores lo asocian a un mal manejo de la muestra durante su preparación, adicionalmente los hidrocarburos en estudio presentan un alto grado de maduración y biodegradación. 


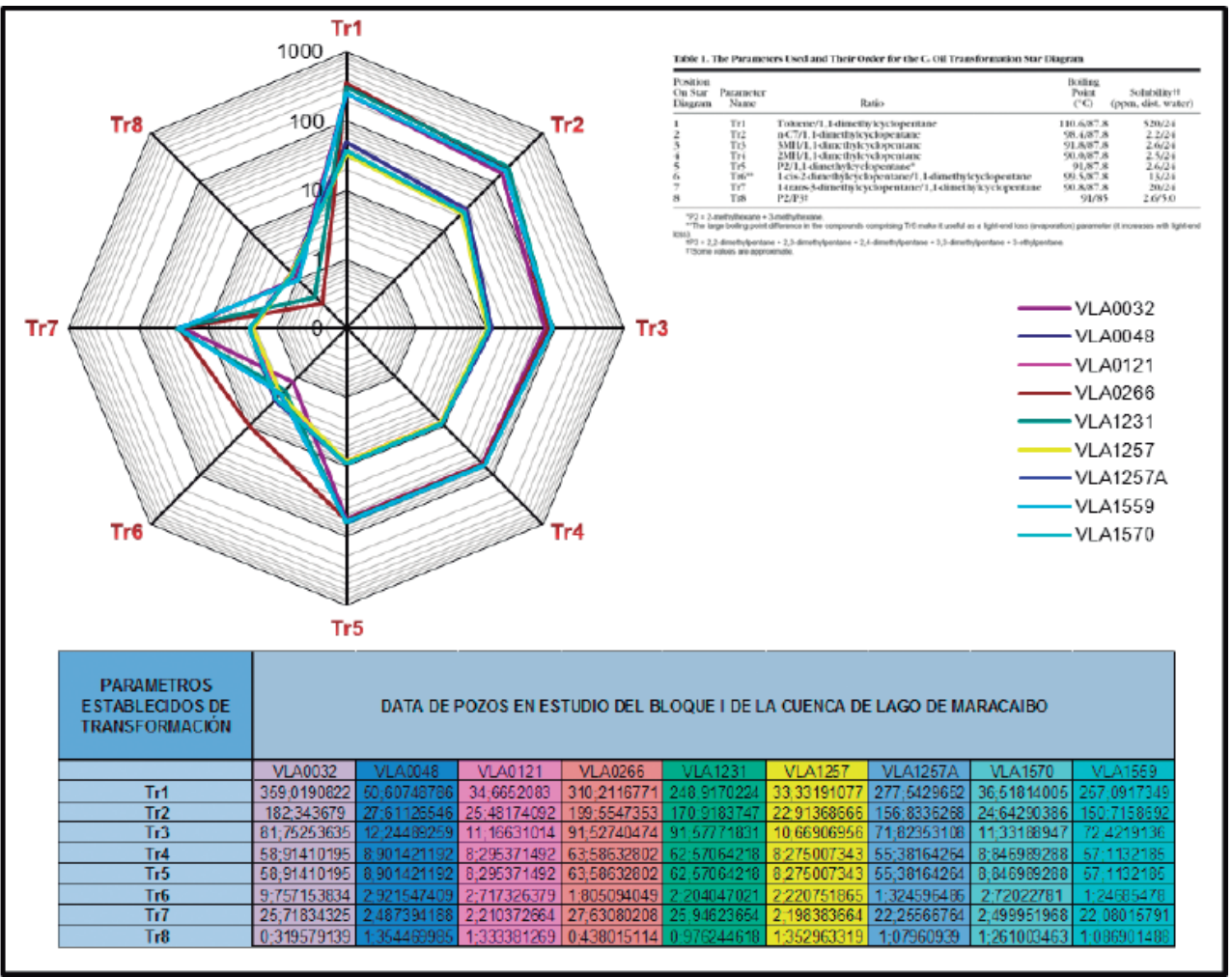

Ilustración 3. Diagrama de estrella de Halpern (1988) para determinar transformación de crudos.

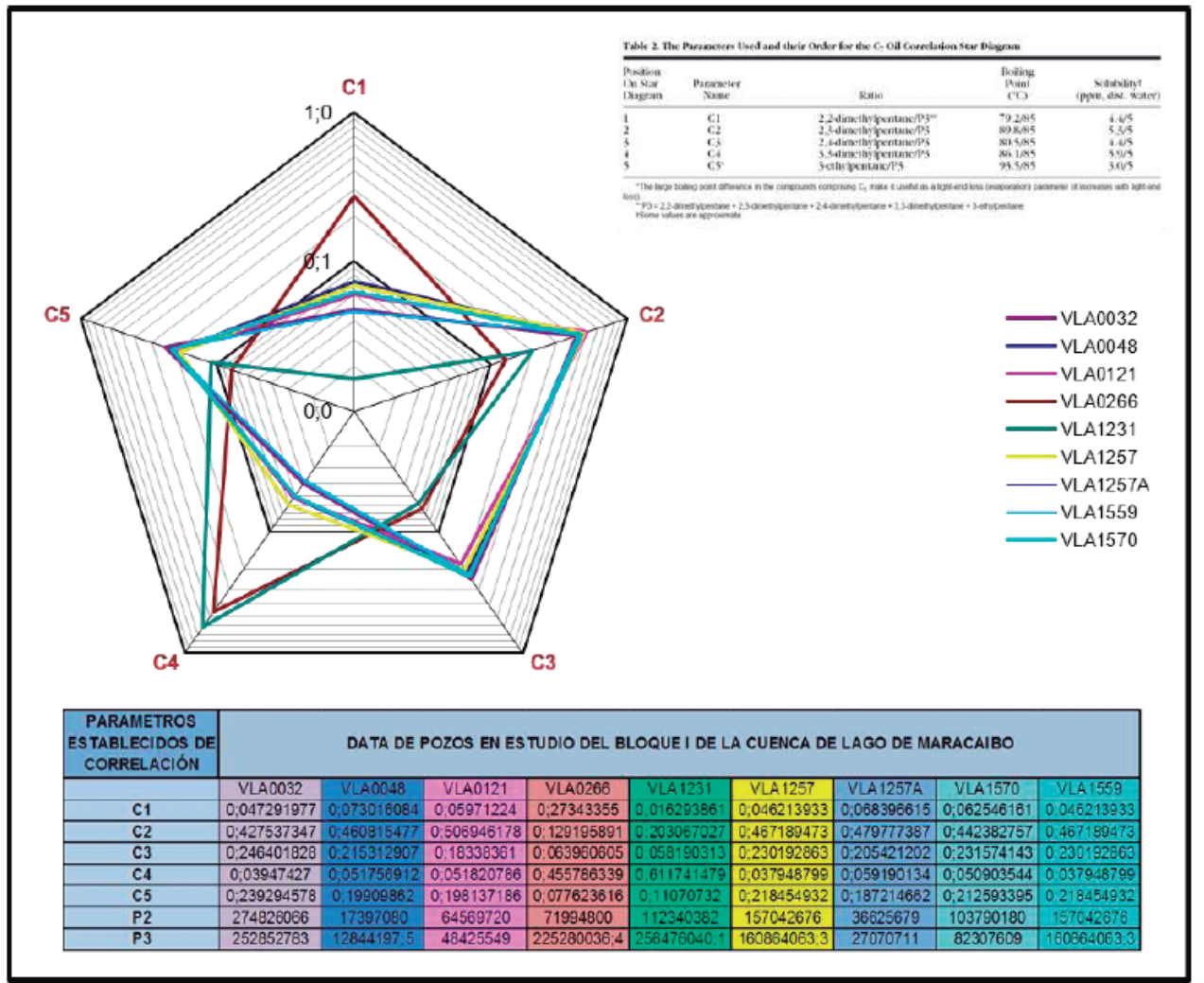

Ilustración 4. Diagrama de estrella de Halpern (1988) para determinar correlación de crudos. 


\section{Diagramas Ternarios para Reconocer Clases Geoquímicas de Crudos}

Los resultados obtenidos en los análisis de la composición de los crudos en base a su solubilidad (S.A.R.A) y el diagrama ternario derivado de ellos, son mostrados en la ilustración 5 se observa que la agrupación de datos correspondiente a todos los crudos de los pozos estudiados, entran en su gran mayoría en la clasificación de crudos normales, según lo referencian, La composición SARA para las arenas en estudio, son principalmente de origen parafínico (saturados), y se pude indicar que estos crudos por estar a mayor profundidad presentan un mayor nivel de madurez que los crudos de las arenas más someras de áreas adyacentes (Meneses et al, 2017).

Diagrama Ternario SARA de Grudos Seguin Tissot $y$ Welte, 1984)

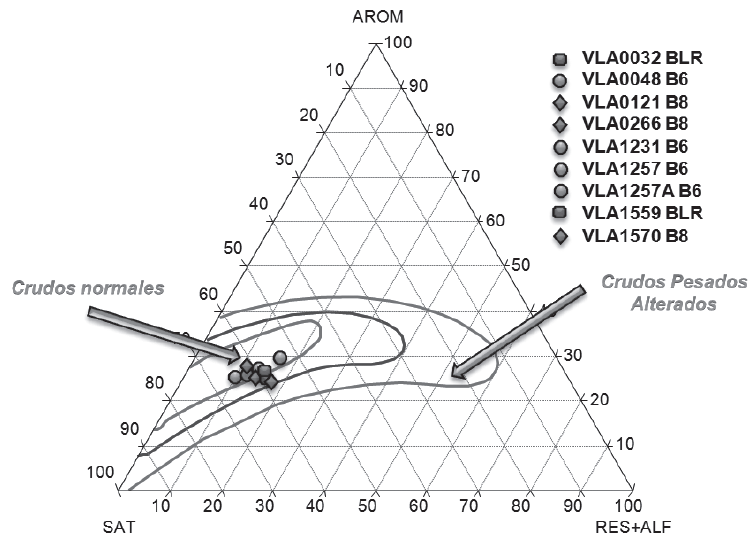

\begin{tabular}{|c|c|c|c|c|c|}
\hline POZOS & \% SATURADOS & \% AROMÁTICOS & \% RESINAS & \% ASFALTENOS & $\%$ RES+ASF \\
\hline VLA 0032 & $59 ; 55$ & $24 ; 17$ & $15 ; 69$ & $0 ; 59$ & $16 ; 28$ \\
\hline VLA 0266 & $59 ; 18$ & $24 ; 62$ & $15 ; 90$ & $0 ; 30$ & $16 ; 20$ \\
\hline VLA 1231 & $59 ; 70$ & $26 ; 69$ & $13 ; 23$ & $0 ; 38$ & $13 ; 61$ \\
\hline VLA 1257 & $54 ; 36$ & $28 ; 68$ & $16 ; 35$ & $0 ; 61$ & $16 ; 96$ \\
\hline VLA 1559 & $59 ; 60$ & $25 ; 13$ & $14 ; 81$ & $0 ; 47$ & $15 ; 28$ \\
\hline VLA0048 & $62 ; 52$ & $25 ; 05$ & $12 ; 2$ & $0 ; 23$ & $12 ; 43$ \\
\hline VLA0121 & $61 ; 55$ & $27 ; 06$ & $11 ; 12$ & $0 ; 27$ & $11 ; 39$ \\
\hline VLA1257A & $65 ; 29$ & $24 ; 55$ & $10 ; 06$ & $0 ; 1$ & $10 ; 16$ \\
\hline VLA1570 & $61 ; 33$ & $24 ; 35$ & $13 ; 79$ & $0 ; 53$ & $14 ; 32$ \\
\hline
\end{tabular}

Ilustración 5. Diagrama ternario de las fracciones SARA (saturados, aromáticos, resinas y asfaltenos) con información de los crudos del área de estudio.

\section{Relación entre Pr/n-C17 y F/n-C18}

En términos generales, las relaciones entre isoprenoides son parámetros que definen el tipo de aporte de materia orgánica ya que una relación de $\mathrm{Pr} / \mathrm{Ph}$ inferior a 1 indica ambientes de depósitos anóxicos y por el contrario, relaciones de $\mathrm{Pr} / \mathrm{Ph}$ superiores a 1 indican ambientes óxicos o disóxicos. Si estas relaciones superan el valor de 3 ya se considera un claro aporte terrestre. Al analizar los datos, se observa que todas las muestras presentan una relación $\mathrm{Pr} / \mathrm{Ph}$ menor a 1. De acuerdo a esta relación, se puede inferir un origen de la materia orgánica de tipo marino con algo de aporte de materia orgánica terrestre.

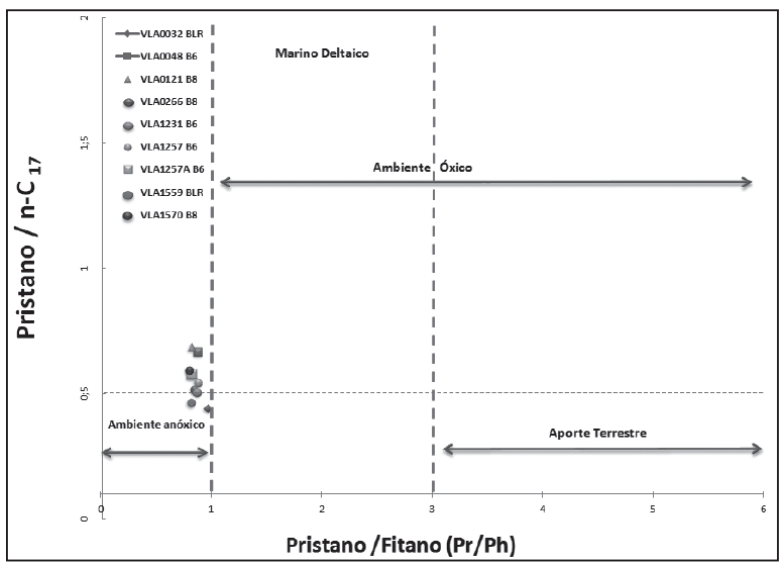

Ilustración 6. Relaciones $\mathrm{Pr} / \mathrm{Ph} v s$. $\mathrm{Pr} / \mathrm{nC} 17$ de los crudos del área de estudio.

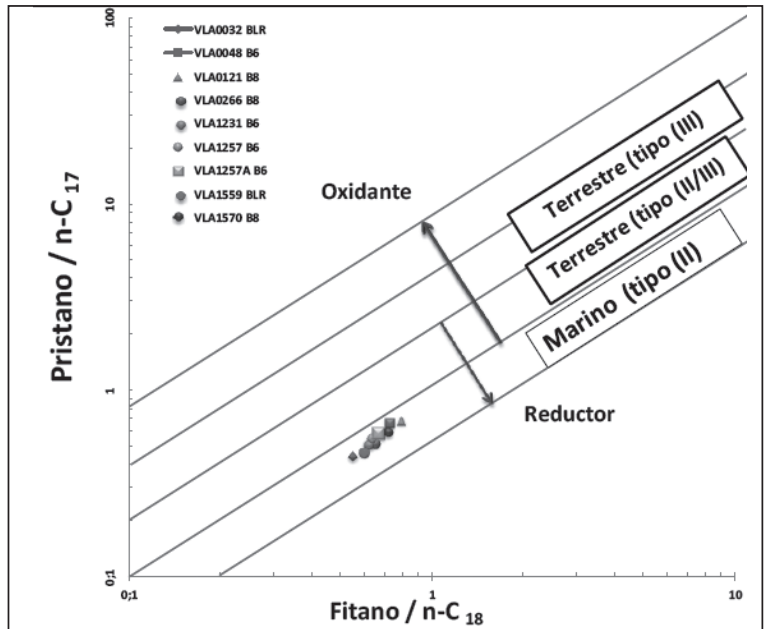

Ilustración 7. Relaciones $\mathrm{Pr} / \mathrm{Ph} v s . \mathrm{Pr} / \mathrm{nC} 17$ de los crudos del área de estudio.

Por otra parte, la relación $\mathrm{Pr} / \mathrm{nC}_{17}$ vs. $\mathrm{Ph} / \mathrm{nC}_{18}$ según Hunt, (1996), muestra también valores inferiores a uno, lo cual es particular de una materia orgánica de tipo marino en condiciones de reducción lo cual se atribuye a un estado de madurez desarrollado, característico de los crudos del área.

La distribución bimodal de n-alcanos revela aporte de materia orgánica marina, y la relación Pristano/Fitano indica condiciones de reducción. Esto se atribuye al avanzado estado de madurez térmica de las muestras. Los marcadores de CPI (Índice Preferencial de Carbono) corroboran que se está en presencia de un crudo maduro con posibles mezclas, según Escobar (2007). 


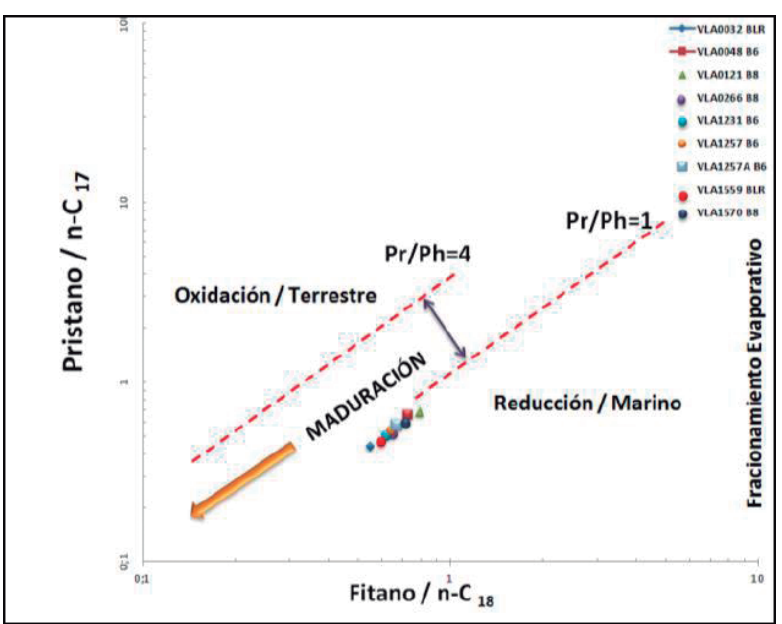

Ilustración 8. Relaciones $\mathrm{Pr} / \mathrm{nC} 17$ vs. $\mathrm{Ph} / \mathrm{nC} 18$ de los crudos del área de estudio.

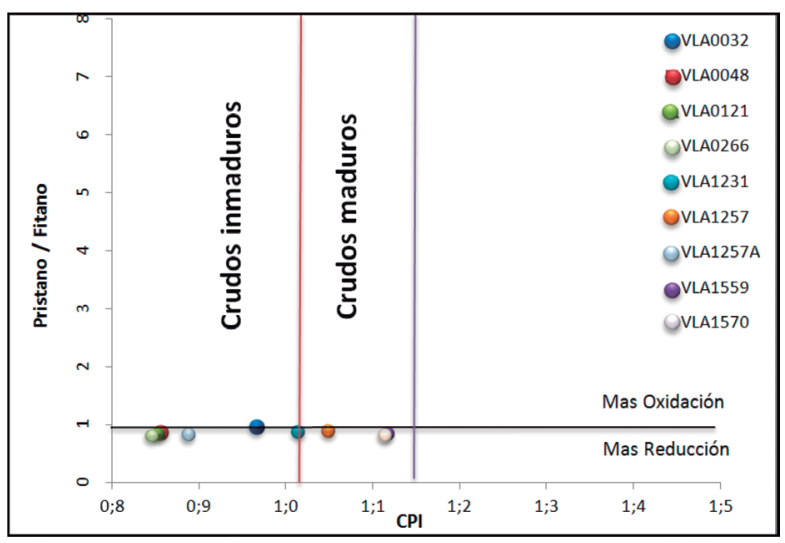

Ilustración 9. Relaciones $\mathrm{Pr} / \mathrm{Ph}$ vs. CPI de los crudos del área de estudio.

\section{Madurez Térmica}

Las relaciones $\mathrm{Ts} / \mathrm{Tm}$ se utilizan con frecuencia para la determinación del grado de madurez. A medida que aumenta el grado de madurez, Tm incrementa mientras que Ts permanece constante. Un aumento en la relación Ts/Tm indicaría un aumento en el grado de evolución (Philp, 1985).

Si nos fijamos en los valores de Ts/Tm presentan valores de 0.47 y 0.55 respectivamente. Caracterizando a las muestras como relativamente maduras. No obstante, Ts y Tm también dependen del tipo de materia orgánica, Ts y $\mathrm{Tm}$ son biomarcadores que se caracterizan por su resistencia frente a la biodegradación.

Es por ello que la aplicación de la relación Ts/Tm como indicador de madurez hay que utilizarla con prudencia, ya que los petróleos que presentan las relaciones más altas pueden haber sufrido algún tipo de biodegradación, mientras que las relaciones anormalmente bajas pueden mostrar una procedencia de una roca madre carbonática.

Los crudos del área de interés en estudio presenta un rango de madurez amplio donde los parámetros Ts $(T s+T m)$ varían entre los valores de 0,41 a 0,55 . En el gráfico 34 , se observa con la relación Ts (Ts + $\mathrm{Tm})$ presenta una tendencia definida al incrementar la gravedad $\mathrm{API}^{\circ}$ de los crudos, la relación anómala de madurez Vs Gravedad $\mathrm{API}^{\circ}$ que presentan los crudos de estudio, probablemente es el resultado de alguna causa de alteración de tipo fraccionamiento - migración como se observa en resultados anteriores, y estos también una buena madurez térmica de los hidrocarburos a medida que los valores van en crecimiento.

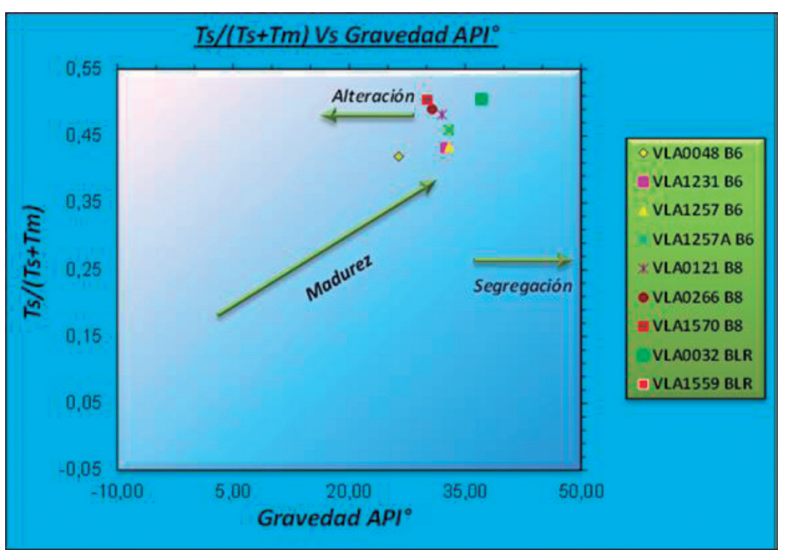

Ilustración 10. Relación de los Hópanos con la diagénesis de los crudos en el área de estudio.

Integrando la información de los crudos relacionados, puede observarse que los crudos presentan buena de madurez inducidos por el sepultamiento gradual de las rocas generadoras de la Formación La Luna, Gómez y Urdaneta (2013).

De esta manera, una importante cantidad de crudos livianos en la Cuenca de Maracaibo, han sido generados en una etapa relativamente tardía de generación. Sin embargo, puede observarse que la biodegradación ha afectado también algunos crudos livianos específicamente a los de las Arenas B del área de estudio.

Otro parámetro utilizado para determinar el nivel de madurez es el índice de metilfenantreno (MPI-1). Este índice se basa en la distribución del metilfenantreno y sus homólogos metilados. Éstos pueden derivar de esteroides y triterpenoides originalmente presentes en el material biológico de partida; o bien pueden originarse a partir de reacciones de metilación del fenantreno. Los isómeros que son más estables desde el punto de vista 
termodinámico son, $\operatorname{los} 2,3$ metilfenantreno, frente a 1 , 9 metilfenantreno, según Peters et al., (2005). También sirven para estimar el porcentaje de reflectancia de vitrinita calculado, ya que se ha observado que guardan una relación lineal, según Peters et al., (2005). Tal y como muestran la siguiente ilustración, todas las muestras estudiadas tienen un valor de vitrinita de aproximadamente igual 1, con lo cual son muestras maduras y que todas ellas están dentro de la ventana de generación de petróleo.

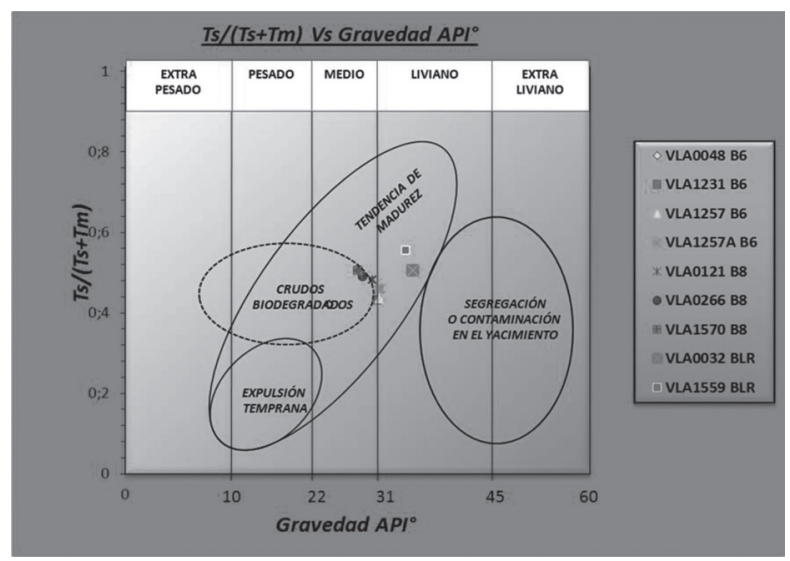

Ilustración 11. Relación generalizada entre la Gravedad API y la madurez de los crudos del área de estudio, utilizando el parámetro Ts / $(\mathrm{Ts}+\mathrm{Tm})$.

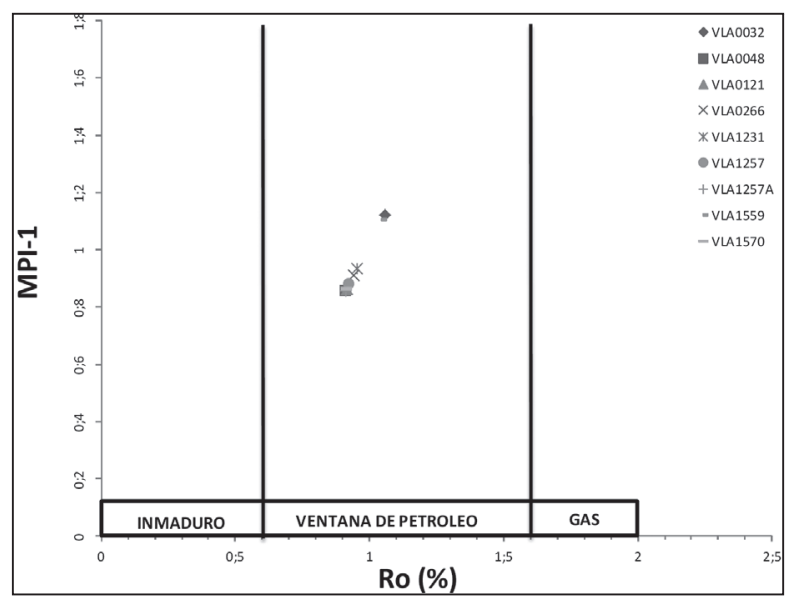

Ilustración 12. Relación entre el índice de metil-fenantreno y el valor de vitrinita.

\section{Conclusiones y Recomendaciones}

- A través del $\mathrm{C}_{7} \mathrm{OCSD}$, se logró diferenciar la huella digital del crudo en cada una de las muestras provenientes del área de interés, encontrándose diferencias moleculares entre ellas.

- A través de $\mathrm{C}_{7} \mathrm{OTSD}$, se muestra un alto porcentaje de tolueno, lo que indica que los crudos no han sido alterados por lavado por aguas, indicando una biodegradación progresiva.

- Los crudos de interés muestran una buena maduración, acotando también que éstos tienen aporte de materia orgánica terrestre.

- El compuesto MDBT muestra una forma escalonada hacia la derecha, indicando origen de crudo siliciclásticos.

- El valor serosidad vs. Pristano/ Fitano es $>1$ lo cual indica origen marino.

- El análisis de los hopanoides indican abundancia precursora de origen marino.

- La relación Tolueno/n-Heptano evidencia que nuestros crudos fueron afectados por fraccionamiento evaporativo.

- La aplicación de la geoquímica orgánica como una herramienta alterna a las tradicionales PLT y SPT, permite una aproximación efectiva y de bajo costo en el seguimiento de producción conjunta de pozos del área, eliminando la intervención mecánica del pozo. Se debe revisar el estudio geologico para validar si existen fallas que impidan la mezclas de crudos en los Yacimientos.

- Se debería extender el estudio geoquímica a otras áreas con el propósito de tener un mayor desarrollo del campo.

- Tomar muestras puras de los Yacimientos para caracterizar y determinar posibles áreas de coalescencias.

- Se recomienda calibrar con los resultados obtenidos en el estudio y confirmar el modelo geológico de la zona.

- Es recomendable realizar estudio isotópico de gases para complementar y tener una mayor caracterización de los Yacimientos.

- Se recomienda realizar estudios Quimioestratigraficos para dar mayor robustez a este estudio.

\section{Referencias Bibliográficas}

1. Albarède, F., (1995). Introduction to geochemical Modeling. Cambrige University Press., Cambrige, 543 pp.

2. Bello-Montoya, R., Rosales, E., Sánchez-Martín, V., (1986). Estudio geoquímico en muestras de aceite y roca de los campos del área Comalcalco-Villahermosa, etapa I: México D.F., México, Instituto Mexicano del Petróleo, unpublished report, $70 \mathrm{p}$.

3. Briceño, G. (1999). Comparación e interpretación de dos levantamientos sísmicos 3D, bloque I Lago de Maracaibo Tesis. UCV. Caracas. 99p.

4. Boesi T. (1978). Resumen sobre el origen de 
las acumulaciones de hidrocarburos en la Formación Misoa, Área del Lago de Maracaibo. Caracas, MARAVEN, Informe Interno MAR000534. Venezuela.

5. Castro, N. M., \&amp; Mederos, I. J. (2016). Generación de modelo petrofísicoestratigráfico del Campo Chimire, Venezuela. Revista Fuentes, 14(1), 89-101

6. Escobar, M. (2008). Fundamentos de la Geoquímica del petróleo, curso dictado en XI congreso latinoamericano de Geoquímica orgánica, Porlamar Venezuela, 129 pp.

7. Figueira, J. N., Simão, R. A., Soares, B. G., \&amp; Lucas, E. F. (2017). The influence of chemicals on asphaltenes precipitation: a comparison between atomic force microscopy and near infrared techniques. Revista Fuentes, 15(1), 7-17.

8. Gallango O., Chin-A-Lien M. y Talukdar S. (1984). Estudio Geoquímico Regional de la Cuenca de Maracaibo. Los Teques, INTEVEP, INT00907, 84. Venezuela.

9. Guardia, V. M. D., Torres, M. C., Arenas, C. E. V., Castro, R. H., Toro, G. M., \&amp; Mendoza, O. B. (2011). Análisis de riesgo y simulación de monte carlo en la valoración de proyectosaplicación en la industria de los hidrocarburos. Revista Fuentes, 9(2).

10. Guerrero-Martin, C. A., Montes-Páez, E., de Oliveira, K., Cristina, M., Campos, J., \&amp; Lucas, E. F. (2018, June). Calculating Asphaltenes Precipitation Onset Pressure by Using Cardanol as Precipitation Inhibitor: A Strategy to Increment the Oil Well Production. In SPE Trinidad and Tobago Section Energy Resources Conference. Society of Petroleum Engineers.

11. Halpern, H. (1995) Development and application of light - hidrocarbon - based start diagrams, AAPG Bulletin. Vol 6, $801-815$

12. Hunt, J.M. (1996). Petroleum Geochemistry and Geology. W.H. Freeman and Company. New York. p. 743.

13. Krauskopt, K.B., (1982). Introduction to geochemistry. International Student Edition, McGraw-Hill, Singapore, 617 pp.

14. Kaufman, R.L.; Ahmed, A.S. and Hempkins W.B. (1987). A new technique for the analysis of commingled oil and its application to production allocation calculations. Proceedings Indonesian Petroleum Association, sixteenth annual convention, October 1987.
15. Martin, C. A. G., \&amp; Páez, E. M. (2017). Efeito da salinidade na tensão interfacial do sistema óleo/agua em condições isobáricas e incremento gradual da temperatura. Revista Fuentes, 15(2), 117-124.

16. Martín, C. A. G., García, R. E. P., Niño, J. C. L., \&amp; Lombana, H. B. (2014). Optimización de la metodología para el cálculo de porosidad a través de saturación de fluidos. Revista Fuentes, 12(2).

17. Meneses, A. F. O., Moreno, L. F. C., \&amp; Plata, J. A. R. (2017). Metodología experimental para la estimación de permeabilidades relativas en dos y tres fases por medio de ajuste histórico. Revista Fuentes, 15(1), 75-85.

18. Nakasone L. Miriam O. (1999). Aplicación de la geoquímica orgánica de producción sobre el bloque I, segregación Lagomar, Lago de Maracaibo. Tesis de grado magister Scientiarum (LUZ). Maracaibo - Venezuela.

19. Navas, D. F. G., Suarez, J. P. O., \&amp; Mejía, J. A. P. (2017). Ajuste fino de correlaciones PVT para algunos crudos colombianos. Fuentes: El reventón energético, 15(2), 57-70.

20. Peter et al. (2005). The biomarker guide, volumen 2: biomarkers and isotopes in the petroleum exploraron and earth history. New York, Cambridge University press 1-700 pp.

21. Peters, K. and Moldowan, J. (1993). The biomarker guide: Interpreting molecular fossil in petroleum and ancient sediments. Prentice Hall, London. p. 363.

22. Philp, P. R. (1985). Biológica markers in fossil fuel production : mass spectrometry reviews. Vol 4. 1-54 pp.

23. Quiroga, A., Rodrigo, L., (1990). Curso Sinóptico de Geoquímica del Petróleo, Revista Técnica de Yacimientos Petrolíferos Fiscales Bolivianos. Santa Cruz - Bolivia.

24. Romero, M. (2007). Caracterización Geoquímica de los Yacimientos C-4 VLA-031 y C-5 VLA031 mediante geoquímica de producción. Bloque I, U.E Lagomar. Tesis-1033, Venezuela.

25. S.A. Consultores CSC (1994). Estudio Geoquímico de los crudos de las areniscas del Eoceno C y de la Formación La Rosa (Mioceno) de la Cuenca de Maracaibo. Venezuela.

26. Talukdar, S. y Marcano, F. (1994). Petroleum Systems of the Maracaibo Basin, Venezuela. En: Magoon, L. y Dow, W.: "The Petroleum System- From Source to Trap". AAPG Memoir 60; 1st. Ed. (Tulsa): 463-482. 
27. Thompson, K (1988). Gas - condensa te migraron and oíl fractionation in deltaic systems, Marine and petroleum geologo. Vol 5. 237246 pp.

28. Tissot, B and Welte D (1984). Petroleum Formation and ocurrence. 2nd edition. New York, Sprenger-Verlog, 660-669 pp.

29. Waples DW (1985). Geochemistry in petroleum exploration. International Human Resources Development Corporation. Boston, p. 232.

Recepción: 06 de septiembre de 2018

Aceptación: 20 de junio de 2019 